\title{
Desenvolvimento e avaliação de filme antimicrobiano aromatizado para aplicação em massa de pastel
}

\author{
Development and evaluation of antimicrobial and flavored film for using on pastry dough
}

\author{
Allan Robledo Fialho e Moraes ${ }^{\mathrm{I}}$ Márcia Cristina Teixeira Ribeiro Vidigal ${ }^{\mathrm{I}}$ \\ Nilda de Fátima Ferreira Soares ${ }^{*}$ Luana Pereira de Moraes $^{\mathrm{II}}$ Nathália Ramos de Melo ${ }^{\mathrm{III}}$ \\ Maria Paula Junqueira Gonçalves ${ }^{\mathrm{IV}}$
}

\section{RESUMO}

\begin{abstract}
A indústria de alimentos, buscando atender à crescente demanda dos consumidores, vem desenvolvendo embalagens ativas para proporcionar qualidade e segurança aos produtos acondicionados. Este trabalho objetivou desenvolver e avaliar a aplicação de filmes ativos aromatizados em contato com a massa de pastel. Os filmes foram preparados pelo método casting, adicionados de ácido sórbico e aroma de pizza e avaliados in vitro frente ao microrganismo Penicillium sp. Também foram estudadas suas propriedades mecânicas, migração de ácido sórbico, avaliação sensorial do produto $e$ análise microbiológica in vivo. Os filmes ativos apresentaram atividade antimicrobiana tanto in vitro como no alimento. A caracterização mecânica mostrou que os filmes ativos aromatizados apresentaram valores de carga máxima na ruptura, inferiores ao filme controle e, durante a migração, a adição de aroma contribuiu para uma maior liberação do ácido sórbico. Além disso, as massas de pastel em contato com os filmes ativos aromatizados apresentaram melhores resultados sensoriais.
\end{abstract}

Palavras-chave: filme ativo, ácido sórbico, segurança alimentar, Penicillium sp.

\section{ABSTRACT}

The food industry, trying to meet growing consumer demand, is developing active packaging to provide quality and safety for packed food. This research aimed to develop and evaluate the implementation of active flavored films in contact with pastry dough. The active films were evaluated for in vitro antimicrobial activity against Penicillium sp. The mechanical properties of the films, the sorbic acid migration, sensory and in vivo microbiological analyses were also tested. The active films showed better results for in vitro and in vivo microbiological analyses when compared with the film with the sorbic acid incorporated directly on the pastry dough. The incorporation of sorbic acid and flavor affected the mechanical properties of the active films compared to the control film. The flavor addition provided a larger migration of sorbic acid from the film to the pastry dough. Besides the pastry dough packed in the active films showed better sensory results.

Key words: active packaging, sorbic acid, food safety, Penicillium sp.

\section{INTRODUÇÃO}

As indústrias de alimentos têm aumentado e diversificado os alimentos processados na tentativa de suprir o mercado crescente. Nos últimos anos, temse ampliado pesquisas na área de embalagem e uma nova linha tecnológica está em destaque: as embalagens ativas. Essas embalagens interagem com o produto acondicionado objetivando melhorar sensorialmente e/ou estender a vida de prateleira dos produtos (SOARES et al., 2009).

Os filmes ativos com função aromatizante e antimicrobiana baseiam-se na incorporação de aromas e conservantes alimentares na estrutura do polímero durante o processo de produção de filmes. Eles

\footnotetext{
'Departamento de Tecnologia de Alimentos, Universidade Federal de Viçosa (UFV), 36570-000, Viçosa, MG, Brasil. E-mail: nfsores@ufv.br.*Autor para correspondência.

"Departamento de Ciência e Tecnologia de Alimentos, Universidade Estadual de Campinas (UNICAMP), Campinas, SP, Brasil.

IIIDepartamento de Engenharia de Agronegócios, Universidade Federal Fluminense (UFF), Volta Redonda, RJ, Brasil.

${ }^{\text {IV }}$ Departamento de Ciencia y Tecnología de Alimentos, Universidad de Santiago de Chile (USACH), Chile.
} 
objetivam reduzir, inibir ou retardar o crescimento da microbiota presente, principalmente na superfície do alimento embalado, onde a maior parte das reações de deteriorização ocorre, além de adicionar características aromáticas ao alimento (SOARES et al., 2009). O aroma constitui uma propriedade sensorial extremamente importante e é um dos principais critérios de aceitação do consumidor (MINIM, 2006). Dentre os conservantes utilizados em alimentos, os ácidos orgânicos merecem destaque por possuírem baixa interferência no sabor e baixo nível de toxicidade (SILVEIRA et al., 2007).

Por ser uma tecnologia com boas perspectivas na indústria de alimentos, o objetivo deste trabalho foi desenvolver filmes ativos antimicrobianos aromatizados para aplicação em massa de pastel, avaliar suas propriedades mecânicas, a migração do ácido sórbico para a massa, a eficiência microbiológica in vitro e in vivo e a sua aceitabilidade sensorial.

\section{MATERIAL E MÉTODOS}

Produção dos filmes ativos

Os filmes de base celulósica foram produzidos pelo método casting, segundo SILVEIRA et al. (2007). À solução filmogênica foi adicionado ácido sórbico (Merck) nas concentrações $0 \%$ e $7 \%$ e aroma de pizza (IFF) nas concentrações de $0 \%, 15 \%$ e $30 \%$ em relação à resina. Os tratamentos foram: filme celulósico sem adição de ácido sórbico e aroma (controle) (Tratamento 1); filme celulósico incorporado com $7 \%$ de ácido sórbico (Tratamento 2); filme celulósico incorporado com $7 \%$ de ácido sórbico e $15 \%$ de aroma de pizza (Tratamento 3) e filme celulósico incorporado com $7 \%$ de ácido sórbico e $30 \%$ de aroma de pizza (Tratamento 4). Os filmes foram submetidos à esterilização em luz UV/nm (Prodicil, 110V, 254nm) por cinco minutos, embalados em filme de alta barreira e armazenados em temperatura ambiente $\left(23 \pm 2^{\circ} \mathrm{C}\right)$ para posterior avaliação.

\section{Avaliação in vitro dos filmes}

Sobre Ágar Batata Dextrose (Himedia) solidificado em placa de Petri, foi semeado $0,1 \mathrm{~mL}$ de uma suspensão $10^{6} \mathrm{UFCmL}^{-1}$ do microrganismo Penicillium spp. e, sobre este, foi colocado, assepticamente, no centro, um disco do filme produzido $(\varnothing 2,1 \mathrm{~cm})$ (APPENDINI \& HOTCHKISS, 2002). As placas foram incubadas a $25^{\circ} \mathrm{C}$ por cinco dias e avaliadas quanto à formação de halo de inibição por meio de sua medida.

Análises das propriedades mecânicas dos filmes ativos Os filmes foram avaliados quanto à carga máxima na ruptura $(\mathrm{N})$ e ao alongamento na carga máxima (\%) (ASTM-D882-00, 2001), utilizando o Aparelho Universal de Testes Mecânicos (Instronmodelo 3367, Estados Unidos), com célula de carga de $1 \mathrm{kN}$, velocidade de tração de $20 \mathrm{~mm} \cdot \mathrm{min}^{-1}$ e distância entre garras de $125 \mathrm{~mm}$.

Eficiência antimicrobiana dos filmes ativos quando em contato com massa de pastel

As massas de pastel foram produzidas por empresa local. À farinha de trigo foi misturada gordura vegetal, cloreto de sódio, glutamato monossódico e água. Como controle, foi produzida uma massa contendo $0,18 \%$ de sorbato de potássio, conforme utilizado pela empresa. As massas foram cortadas com $10 \mathrm{~cm}$ de diâmetro, intercaladas em filmes de polietileno de baixa densidade (PEBD) e conduzidas ao Laboratório de Embalagem-UFV, sob refrigeração. As massas sem conservantes foram intercaladas com os filmes ativos e a massa contendo sorbato de potássio com o filme controle (sem antimicrobiano e aroma) e armazenadas sob refrigeração $\left(8 \pm 1^{\circ} \mathrm{C}\right)$ por 40 dias. As massas foram analisadas quanto à contagem de psicrotróficos, fungos filamentosos e leveduras e Salmonella nos tempos 0, 20 e 40 dias de estocagem, conforme VANDERZANT \& SPLITTSTOESSER, 1992.

Migração do Ácido Sórbico do Filme para a Massa de Pastel

As massas de pastel foram analisadas após 1, 2, 3, 4, 6, 8, 12, 20, 30 e 40 dias de armazenamento. Para extração do ácido sórbico, foi utilizada a metodologia descrita por TFOUNI E TOLEDO (2002), com algumas modificações. A dois gramas de massa de pastel, foram adicionados 31,6g de etanol (40mL; F. Maia, $100 \%$ de pureza) e agitados por 3 horas (agitador Fisatom, modelo 752, Brasil) à temperatura ambiente. $\mathrm{O}$ volume de etanol evaporado foi corrigido e o volume da mistura completado para $100 \mathrm{~mL}$ com água deionizada (Milli-Q Gradient, Millipore, Irlanda). A amostra foi novamente agitada por 15 minutos. O sobrenadante foi removido, filtrado (filtro celulósico de $0,22 \mu \mathrm{m}$ de poro) e armazenado em frasco âmbar à temperatura de refrigeração $\left(8 \pm 1^{\circ} \mathrm{C}\right)$.

A quantificação do ácido sórbico migrado para a massa de pastel foi realizada por análise de Cromatografia Líquida de Alta Eficiência (CLAE) (cromatógrafo SHIMADZU-modelo 10 AVP, coluna Shim-pack ODS - 4,6 $\mu \mathrm{m}, 4,6 \times 150 \mathrm{~mm}$, Japão), segundo CHOI et al. (2001). Foram analisados $20 \mu \mathrm{L}$ da amostra, tendo como fase móvel $81 \%$ de água, $17 \%$ de acetonitrila e $2 \%$ de tampão fosfato de amônio $0,005 \mathrm{M}(\mathrm{pH} \mathrm{4,2}$ ajustado com ácido acético glacial), fluxo de $1 \mathrm{~mL} \mathrm{~min}^{-1}$, e a detecção realizada em 260nm. A quantificação foi realizada por comparação dos resultados 
cromatográficos das amostras com a curva analítica gerada.

Para a geração da curva analítica, foi preparada solução estoque de ácido sórbico (Vetec, $100 \%$ pureza) na concentração 500ppm e, a partir desta, foram feitas diluições sucessivas nas concentrações $250,150,50,25$ e $0 \mu \mathrm{LL}^{-1}$. As diluições foram analisadas por CLAE, segundo metodologia descrita anteriormente.

\section{Análise Sensorial}

A aceitabilidade sensorial das massas de pastel foi realizada por 44 consumidores após 40 dias de armazenamento. As massas de pastel fritas foram servidas em cabines individuais, de forma monádica, codificadas com números aleatórios de três dígitos e avaliadas quanto à aceitação em relação ao aroma, sabor e impressão global, utilizando escala hedônica de nove pontos (MINIM, 2006).

\section{Análise Estatística}

Todo o experimento foi realizado com três repetições. Os resultados do teste de difusão em ágar, análise microbiológica e migração foram submetidos à análise descritiva. Os resultados da análise sensorial e das propriedades mecânicas foram submetidos à análise de variância (ANOVA) e teste de comparação de médias Tukey $(\mathrm{P}<0,05)$, utilizando-se o programa estatístico SAS, versão 9.1, licenciado para a Universidade Federal de Viçosa.

\section{RESULTADOS E DISCUSSÕES}

Avaliação in vitro dos filmes

Os filmes incorporados com ácido sórbico apresentaram efeito antimicrobiano, sendo observada a formação de halos de inibição ao redor do filme quando em contato com o fungo Penicillium sp. Os filmes ativos aromatizados apresentaram maior eficiência antimicrobiana (Figura 1), possivelmente, devido à abertura da cadeia polimérica promovida pelo composto, que favoreceu a migração do agente antimicrobiano do filme para o meio. $\mathrm{O}$ aroma pode ter se comportado como um plastificante, ou seja, compostos de baixo peso molecular que reduzem as ligações secundárias entre polímeros através da separação das cadeias poliméricas, promovendo maior mobilidade das macromoléculas (RAHMAN e BRAZEL, 2004).

Propriedades mecânicas dos filmes ativos

A carga máxima na ruptura dos filmes diminuiu significativamente $(\mathrm{P}<0,05)$ com a incorporação de aromatizantes e o filme ativo com $30 \%$ de aroma apresentou maior percentagem de alongamento (Tabela 1). Resultados similares foram encontrados por GOUVEIA et al. (2006) ao avaliarem as propriedades mecânicas de filmes ativos aromatizados. Diante desses resultados, pode-se inferir que o aroma atua como um plastificante, alterando as propriedades mecânicas dos filmes, aumentando sua flexibilidade.

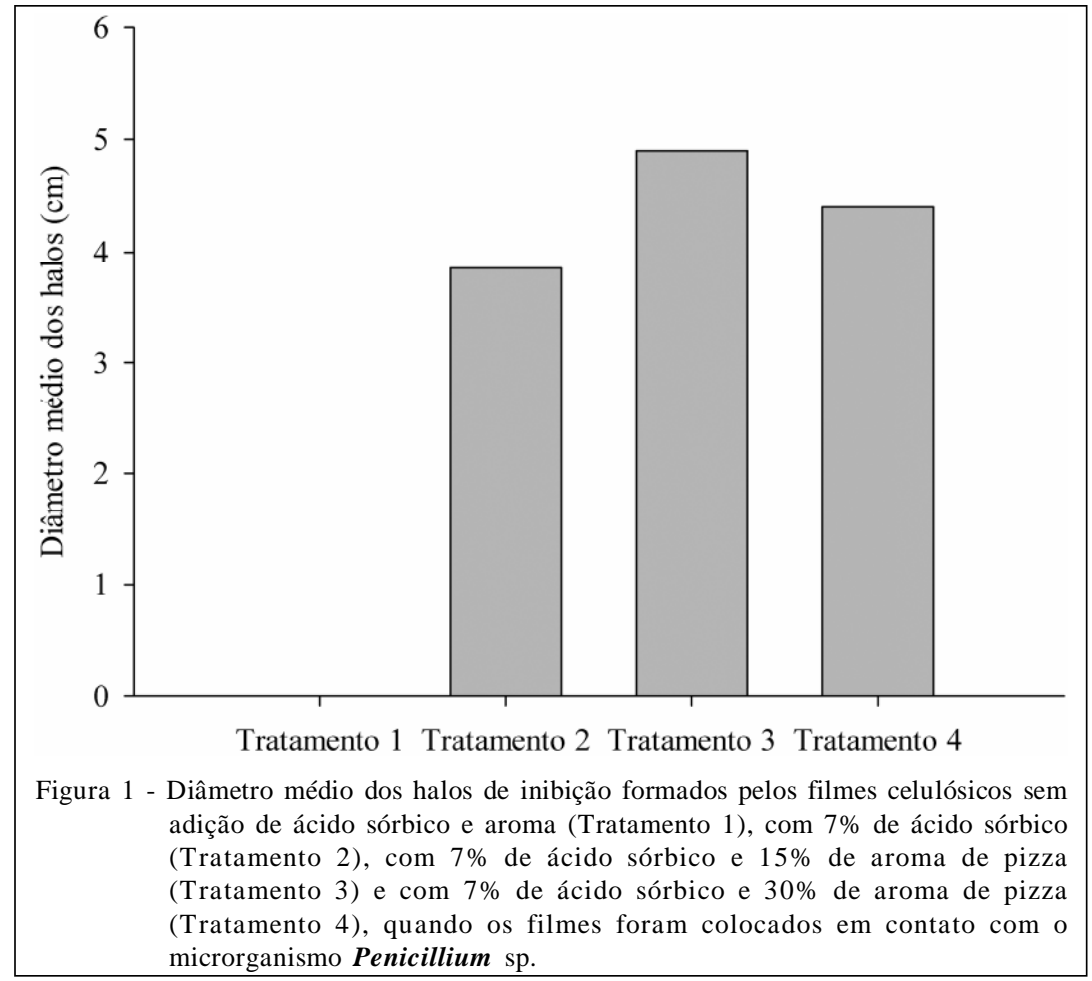

Ciência Rural, v.41, n.3, mar, 2011. 
Tabela 1 - Média das propriedades mecânicas, alongamento (\%) e carga máxima na ruptura (N) dos filmes desenvolvidos.

\begin{tabular}{ccc}
\hline Filme & Alongamento $(\%)$ & Carga máxima na ruptura (N) \\
\hline Controle & $2,9^{\mathrm{b}}$ & $87,8^{\mathrm{a}}$ \\
$7 \%$ Ac. sórbico + 0\% Aroma de pizza & $3,1^{\mathrm{b}}$ & $83,7^{\mathrm{a}}$ \\
$7 \%$ Ac. sórbico + 15\% Aroma de pizza & $3,0^{\mathrm{b}}$ & $49,6^{\mathrm{b}}$ \\
$7 \%$ Ac. sórbico + 30\% Aroma de pizza & $4,7^{\mathrm{a}}$ & $57,4^{\mathrm{b}}$ \\
\hline
\end{tabular}

Médias seguidas pela mesma letra, na mesma coluna, não diferem entre si pelo teste de Tukey a 5\% de probabilidade.

Eficiência antimicrobiana dos filmes em contato com a massa de pastel

As massas de pastel intercaladas com filmes contendo $7 \%$ de ácido sórbico (Tratamento 2) apresentaram menor crescimento microbiano em relação à massa controle. Os resultados deste trabalho corroboram os reportados por SILVEIRAet. al. (2007) e QUATTARA et al. (2000), em que o emprego de filmes antimicrobianos foi mais eficiente do que o uso direto de antimicrobiano nos alimentos, pois o antimicrobiano migra lenta e gradualmente da embalagem para a superfície do alimento, onde mantém-se na concentração necessária para inibir o desenvolvimento de microrganismos. Na maioria dos alimentos, a contaminação microbiana ocorre, em maior intensidade, na sua superfície, onde se requer maior controle efetivo do crescimento microbiano (PADGETT et al., 1998).

Coerentemente com o resultado do teste de difusão em ágar, os filmes incorporados com aroma apresentaram maior eficiência antimicrobiana, possivelmente, devido ao favorecimento da migração do agente antimicrobiano do filme para a massa, havendo assim uma maior ação sobre fungos filamentosos, leveduras e psicrotróficos (Figuras 2 e 3). MORAES et al. (2007) demonstraram que filmes ativos incorporados com ácido sórbico são efetivos contra o crescimento de fungos filamentosos e leveduras em manteigas. As análises microbiológicas para Salmonella, nos tempos 0, 20 e 40 dias de estocagem, apresentaram ausência em $25 \mathrm{~g}$ de massa, estando de acordo com a legislação vigente (BRASIL, 2001).

Migração do ácido sórbico do filme ativo para a massa de pastel

Os filmes incorporados com ácido sórbico e aroma (Tratamentos 3 e 4) apresentaram liberação rápida do antimicrobiano para a massa de pastel até atingir o equilíbrio, ou seja, até o máximo de liberação, que ocorreu aproximadamente no $12^{\circ}$ dia de estocagem (Figura 4). O filme ativo sem aroma (Tratamento 2) liberou o conservante em menor quantidade, apresentando concentrações inferiores até mesmo que o tratamento 1 (massa de pastel comercial), em que o

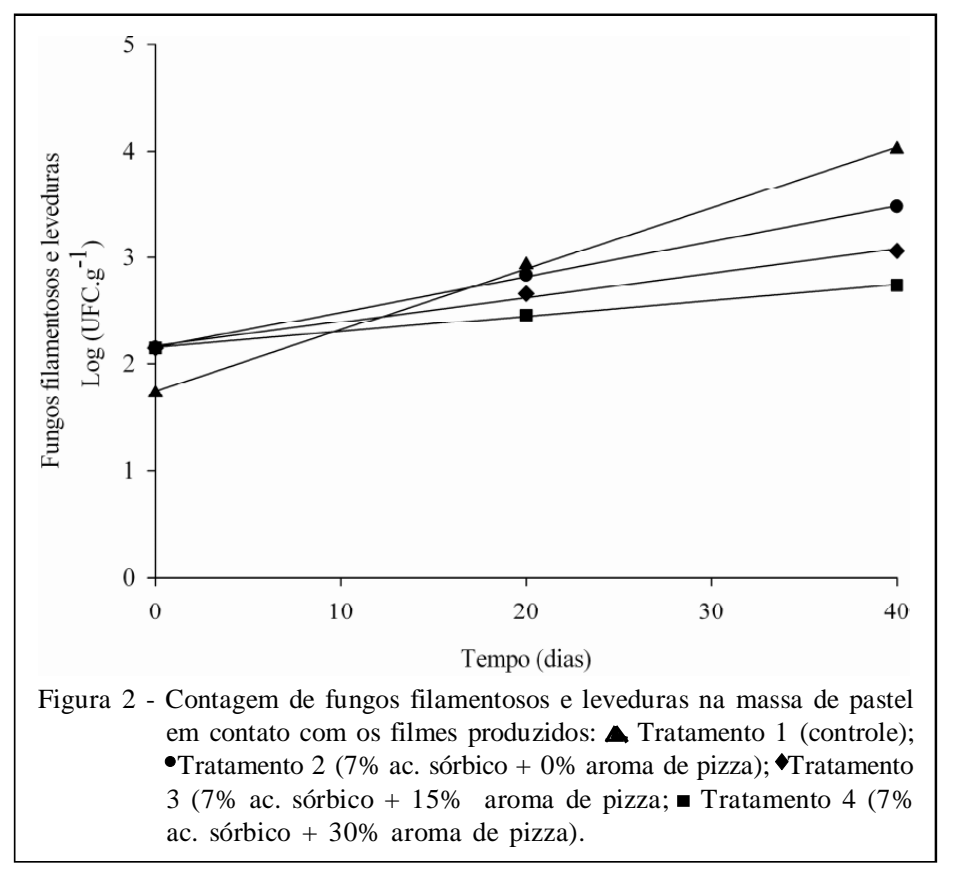

Ciência Rural, v.41, n.3, mar, 2011. 


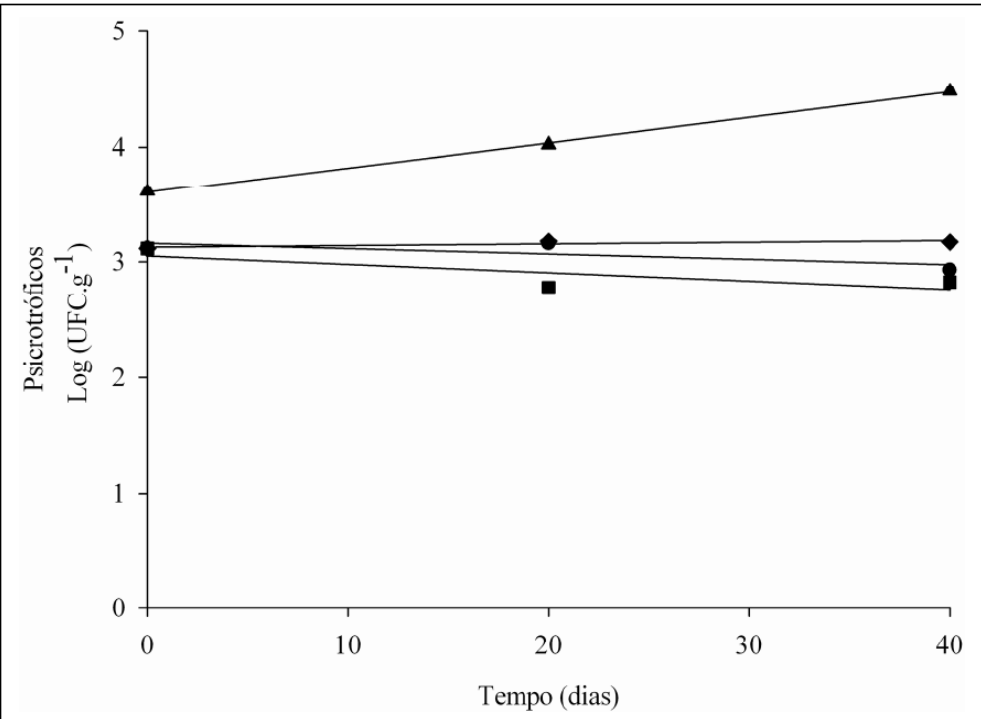

Figura 3 - Contagem de psicrotróficos na massa de pastel em contato com os filmes produzidos: $\mathbf{\Delta}$ Tratamento 1 (controle); $\bullet$ Tratamento 2 (7\% ac. sórbico $+0 \%$ aroma de pizza); $\bullet$ Tratamento $3(7 \%$ ac. sórbico $+15 \%$ aroma de pizza); - Tratamento 4 (7\% ac. sórbico $+30 \%$ aroma de pizza).

ácido sórbico é adicionado diretamente na massa. Esse resultado corrobora o de SILVEIRA et al. (2007). A adição de aroma contribuiu para uma maior liberação do ácido sórbico. Todos os tratamentos apresentaram concentração de ácido sórbico abaixo do limite de $0,1 \%$ permitido pela legislação (BRASIL, 1999). Após o período de estocagem, o tratamento 2 apresentou a menor concentração do conservante $(0,02 \%)$ na massa de pastel, enquanto os tratamentos 3 e 4 apresentaram as maiores concentrações $(0,07 \%)$.

Análise Sensorial

Os resultados da avaliação sensorial dos pastéis, em relação aos atributos aroma, sabor e impressão global apresentaram diferença significativa $(\mathrm{P}<0,05)$ entre os tratamentos (Tabela 2). Em relação ao

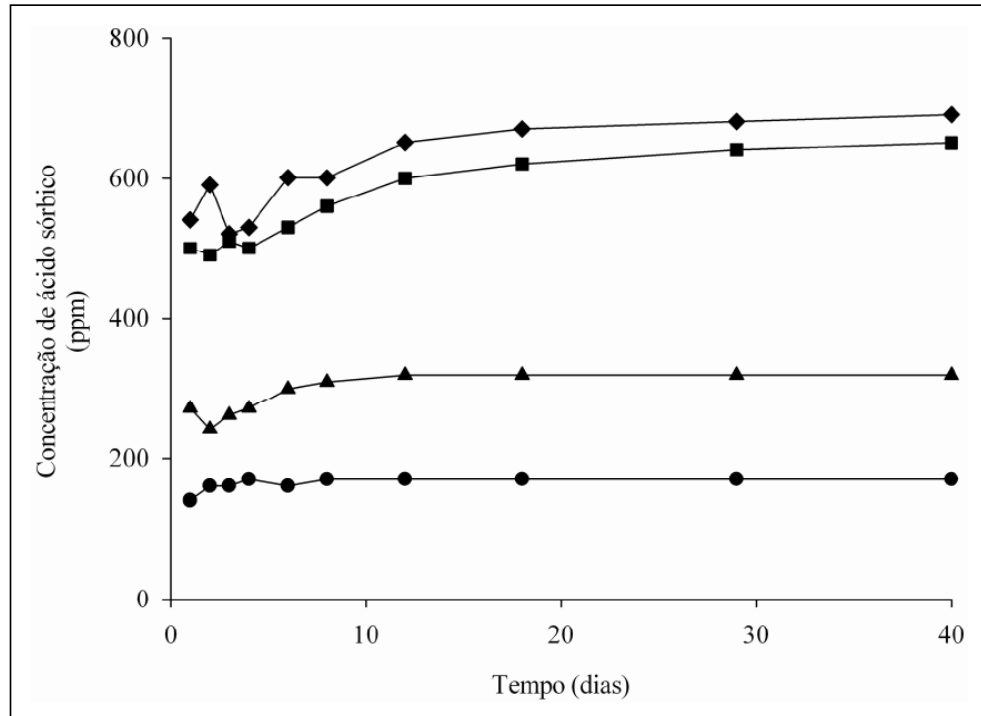

Figura 4 - Curva de migração do ácido sórbico do filme ativo para massa de pastel. $\mathbf{\Delta}$ Tratamento 1 (controle); $\bullet$ Tratamento 2 (7\% ac. sórbico + $0 \%$ aroma de pizza); Tratamento 3 (7\% ac. sórbico $+15 \%$ aroma de pizza); Tratamento 4 (7\% ac. sórbico $+30 \%$ aroma de pizza).

Ciência Rural, v.41, n.3, mar, 2011. 
Tabela 2 - Médias de aceitação das amostras de pastel submetidas a tratamentos com ácido sórbico e aroma de pizza, para os atributos aroma, sabor e impressão global.

\begin{tabular}{|c|c|c|c|}
\hline \multicolumn{4}{|l|}{ Tratamentos } \\
\hline & Aroma & Sabor & Impressão global \\
\hline Controle & $6,64^{\mathrm{b}}$ & $6,34^{\mathrm{b}}$ & $6,50^{\mathrm{b}}$ \\
\hline $7 \%$ Ac. sórbico $+0 \%$ Aroma de pizza & $6,57^{b}$ & $6,29^{b}$ & $6,48^{b}$ \\
\hline $7 \%$ Ac. sórbico $+15 \%$ Aroma de pizza & $7,59^{\mathrm{a}}$ & $7,41^{\mathrm{a}}$ & $7,48^{\mathrm{a}}$ \\
\hline $7 \%$ Ac. sórbico $+30 \%$ Aroma de pizza & $7,27^{\mathrm{ab}}$ & $7,25^{\mathrm{a}}$ & $7,20^{\mathrm{ab}}$ \\
\hline
\end{tabular}

*Médias seguidas de mesma letra em uma mesma coluna não diferem entre si a 5\% de probabilidade pelo teste de TUKEY.

aroma e impressão global, o pastel cuja massa foi separada por filmes com $15 \%$ de aroma de pizza (Tratamento 3) foi a mais aceita, diferindo dos tratamentos sem a adição do aromatizante (Tratamentos 1 e 2). Quanto ao sabor, os pastéis das massas separadas por filmes aromatizados incorporados com $15 \%$ e $30 \%$ de aroma de pizza diferiram estatisticamente dos tratamentos 1 e 2, apresentando maior aceitação $(\mathrm{P}<0,05)$. GOUVEIA et al. (2006) também obtiveram resultados positivos ao avaliarem a aceitação de ricota acondicionada com filme ativo aromatizado com bacon e pizza. Os pastéis das massas com $15 \%$ e $30 \%$ de aroma de pizza foram classificados entre os termos hedônicos gostei moderadamente e gostei muito, indicando que foram bem aceitos.

\section{CONCLUSÃO}

A atividade antimicrobiana dos filmes apresentou-se satisfatória, uma vez que houve inibição do crescimento microbiano nas massas de pastel quando em contato com os filmes ativos. Houve diminuição na carga máxima na ruptura e um aumento na porcentagem de alongamento para os filmes incorporados com aroma, apresentando maior maleabilidade, característica favorável a sua utilização como material de embalagem. A concentração de ácido sórbico na massa, após o período de estocagem, foi menor que o permitido pela legislação. A utilização de filmes ativos antimicrobianos aromatizados apresentase como uma nova tecnologia, que garante a segurança microbiológica, além de reduzir o consumo de aditivo e proporcionar uma maior variedade sensorial do produto.

\section{AGRADECIMENTO}

Fundação de Amparo à Pesquisa do Estado de Minas Gerais (FAPEMIG), Coordenação de Amparo à Pesquisa do Estado de São Paulo (CAPES), Conselho Nacional de Desenvolvimento Científico e Tecnológico (CNPq) e FINEP.

\section{REFERÊNCIAS}

AMERCIAN SOCIETY FOR TESTING AND MATERIALS ASTM. Standard test methods for tensile properties of thin plastic sheet. ASTM D882-00. Philadelphia, 2001. 9p.

APPENDINI, P.; HOTCHKISS, J.H. Review of antimicrobial food packaging. Innovative Food Science \& Emerging Technologies, v.3, p.113-126, 2002.

BRASIL. Resolução n.385, de 5 de agosto de 1999. Regulamento Técnico que Aprova o uso de Aditivos Alimentares, estabelecendo suas funções e seus Limites Máximos para a Categoria de Alimentos 6 - Cereais e Produtos de ou a Base de Cereais. Diário Oficial da União, 1999. Disponível em: <http://www.anvisa.gov.br/legis/resol/385_99.htm>. Acesso em: 10 jan. 2010.

BRASIL. Resolução - RDC n.12, de 2 de janeiro de 2001. Regulamento técnico sobre os padrões microbiológicos para alimentos. Diário Oficial da União, 2001. Disponível em: <http://www.anvisa.gov.br/legis/resol/12_01 rdc.htm>. Acesso em: 05 jan. 2010.

CHOI, J.H. et al. Migration of preservative from antimicrobial polymer coating into water. Food Science Biotechnology, v.10, n.3, p.327-330, 2001.

GOUVEIA, L.E.R. et al. Desenvolvimento e avaliação de filme ativo aromatizado na incorporação de sabor de ricota. Revista do Instituto de Laticínios Cândido Tostes, v.61, n.351, p.138-141, 2006.

MINIM, V.P.R. Análise sensorial: estudos com consumidores. Viçosa: UFV, 2006. V.1, 225p.

MORAES, A.R.F. et al. Desenvolvimento e avaliação de filme ativo antimicrobiano na conservação de manteiga. Ciência e Tecnologia de Alimentos, v.27 (sup1.), p.33-36, 2007.

PADGETT, T. et al. Incorporation of food-grade antimicrobial compounds into biodegradable packaging films. Journal of Food Protection, v.61, n.10, p.1330-1335, 1998. 
QUATTARA, B. et al. Diffusion of acetic and propionic acids from chitosan-based antimicrobial packaging films. Journal of Food Science, v.65, n.5, p.768-773, 2000.

RAHMAN, M.; BRAZEL, C.S. The plasticizer market: an assessment of traditional plasticizers and research trends to meet new challenges. Progress in Polymer Science, v.29, n.12, p.1223-1248, 2004.

SILVEIRA, M.F.A. et al. Active film incorporated with sorbic acid on pastry dough conservation. Food Control, v.18, p.1063-1067, 2007.
SOARES, N.F.F. et al. Novos desenvolvimentos e aplicações em embalagens de alimentos. Revista Ceres, v.56, p.370378, 2009.

TFOUNI, S.A.V.; TOLEDO, M.C.F. Determination of benzoic and sorbic acids in Brazilian food. Food Control, v.13, n.2, p.117-123, 2002.

VANDERZANT, C.; SPliTTSTOESSER, D.F. Compendium of methods for the microbiological examination of foods. 3.ed. Washington DC: American Public Health Association, 1992. 1219p. 